\title{
Norois
}

Environnement, aménagement, société

$218 \mid 2011 / 1$

Campagne : Société, Publicisation, Environnement

\section{Acteurs et agriculture biologique dans la fabrique alternative des espaces : Le cas de l'île-de-France}

Actors and Organic Farming in the alternative territorial Making. Case of Paris Region

Nicolas Boivin et Jean-Baptiste Traversac

\section{OpenEdition}

\section{Journals}

Édition électronique

URL : https://journals.openedition.org/norois/3560

DOI : $10.4000 /$ norois.3560

ISSN : $1760-8546$

Éditeur

Presses universitaires de Rennes

Édition imprimée

Date de publication : 1 juin 2011

Pagination : 41-55

ISBN : 978-2-7535-1360-0

ISSN : 0029-182X

\section{Référence électronique}

Nicolas Boivin et Jean-Baptiste Traversac, « Acteurs et agriculture biologique dans la fabrique alternative des espaces : Le cas de l'île-de-France », Norois [En ligne], 218 | 2011/1, mis en ligne le 30 juin 2013, consulté le 14 janvier 2022. URL : http://journals.openedition.org/norois/3560 ; DOI : https://doi.org/10.4000/norois.3560

Ce document a été généré automatiquement le 14 janvier 2022.

(c) Tous droits réservés 


\section{Acteurs et agriculture biologique dans la fabrique alternative des espaces : Le cas de l'île-de-France}

Actors and Organic Farming in the alternative territorial Making. Case of Paris Region

Nicolas Boivin et Jean-Baptiste Traversac

1 L'agriculture biologique $(\mathrm{AB})$ se veut protectrice des ressources édaphiques et économiques d'un territoire ${ }^{1}$. Compte tenu de cette ambition, elle peut être analysée sous deux perspectives, soit en tant que forme d'agriculture alternative au système d'agriculture dominant, c'est-à-dire un système économique qui se construit en marge, en parallèle ou intégré à l'organisation des systèmes alimentaires locaux et nationaux, soit en tant que forme d'activité économique mettant en exergue des principes éthiques (Clarke et al., 2008). Quatre décennies après la prise de conscience des fortes externalités négatives de l'agriculture productiviste, elle semble être restée en marge $\mathrm{du}$ monde agricole et des pratiques encore largement dominantes de l'agriculture conventionnelle. L'AB se ramifie en filières de distribution, courtes, médianes ou longues, généralement distinctes des filières agroalimentaires conventionnelles qui dominent le marché. Ces traits de caractère la situent parmi les « alternative agri-food networks » (AAFN) (Deverre et Lamine, 2010 ; Higgins et al., 2008). Dans la littérature sur le sujet, l'AB apparait comme une agriculture d'acteurs " politiquement engagés » ou au minimum sensibles à des considérations éthiques. Ces motifs sous-tendent des liens spécifiques entre agriculteurs et consommateurs. Ils impactent également la relation agriculteur-distributeur. Intégrant des fonctions agricoles mais aussi distributives, les agriculteurs se rencontrent à la fois en amont et en aval de la chaîne de création de valeur. Se faisant, ils créent du territoire alternatif tout autant qu'ils participent au renouvellement de l'offre alimentaire hors de l'offre formatée des distributeurs. Les chaînes de marchandises nées de cette pratique agricole, gravitent autour des pôles de distribution parallèles à ceux qui peuvent être qualifiés de «classiques" comme les circuits de grande distribution (Winter, 2003). Toutes les 
régions françaises sont concernées par les transformations dans l'organisation des circuits agroalimentaires provoquées par l'AB ; même si des régions accompagnent avec plus de volontarisme que d'autres le développement des filières bio.

L'Île-de-France compte tenu de sa densité et de l'importance de sa population est un marché considérable pour les producteurs bio. Malgré la présence en son centre de la plus grande agglomération française, les surfaces de terres cultivables dans la région sont importantes. En effet, $50 \%$ des surfaces de cette région sont des terres à vocation agricole qui laissent entrevoir des opportunités nombreuses pour l'installation de producteurs bio. Toutefois il faut noter que cette région s'est fortement spécialisée dans de "grandes» cultures, céréales et oléoprotégineux, très soutenues par la Politique Agricole Commune. Paradoxalement celles-ci ne répondent pas directement à la demande du consommateur francilien en produits frais, qui interpelle plus particulièrement l'AB. Du fait de ces caractéristiques et de son implication dans le développement du bio, l'île-de-France est un exemple particulièrement approprié à l'étude de la structuration du système $\mathrm{AB}$. Il nous a paru opportun d'observer comment dans un tel contexte un système technique innovant peut se développer et participer conjointement au renouvellement de l'offre alimentaire, des pratiques agricoles et des logiques de distribution. Pour la géographie et l'économie régionale ces effets appellent à une lecture des territoires par l'entrée alternative.

3 Dans cet article, le système "agriculture biologique" est à considérer comme l'ensemble des agents, agriculteurs, entreprises de distribution, organisations privées, acteurs publics, qui véhiculent les valeurs d'une agriculture dite durable ${ }^{2}$. Il pose deux principales questions aux analystes de l'évolution des territoires ruraux. Les ancrages territoriaux du système $\mathrm{AB}$ sont-ils réellement alternatifs ou ne sont-ils que des formes renouvelées d'instruments éprouvés de la diffusion des pratiques techniques? Quelles sont les natures des interrelations des acteurs de ces systèmes perçus comme alternatifs? L'impact politique de l'AB réside dans sa capacité à bousculer les paradigmes du schéma dominant. Elle est confrontée aux formes d'efficience technique et économique d'une agriculture céréalière ou maraîchère productiviste. La considération que lui portent consommateurs et citoyens est néanmoins susceptible d'entraîner une nouvelle dynamique agricole et politique par le biais d'acteurs antérieurement présents et actifs dans la fabrique des espaces agricoles et de nouveaux acteurs comme les associations de consommateurs.

Nous proposons une lecture de la reconfiguration du territoire francilien à travers le triptyque acteur, réseau, proximité. Cette approche permet une analyse qui dépasse les schémas classiques de lecture des Systèmes Productifs Locaux. Son interprétation des Systèmes Territoriaux alternatifs place en son centre, les logiques et les stratégies des acteurs. Le territoire n'est plus alors une simple matrice physique mais une forme géographique à part entière, un espace d'actions voulues ou subies. À partir de l'observation de l'espace géographique, les formes territoriales du système se dessinent permettant ainsi la distinction de nœuds et d'interactions en Île-de-France ancrés sur l'AB. Les aspects politiques et techniques de la filière doivent prendre en considération ces territoires réticulaires qui se sont mis en place de façon plus ou moins informelle. La première partie présente le système né de l'AB. Le jeu d'échelle est l'optique choisie pour expliciter les enjeux de ce mode de production. Les rouages «actoriels» du système, la trame des relations bi et multilatérales entre agents économiques, politiques et organisationnels, sont l'objet de la seconde partie. Ils interrogent la 
fonction alternative du système. Enfin, la troisième partie met en exergue le rapport entre les territoires alternatifs et les proximités nés de la réorganisation des espaces agricoles par l'introduction de l'AB.

\section{Le système " agriculture biologique ", quels enjeux locaux et régionaux?}

5 Le système " agriculture biologique » a une morphologie différente selon les territoires. Il est singulier d'une région à l'autre de l'Europe malgré une législation commune au plan européen depuis janvier 2009. En Allemagne, en Suisse, au Danemark, en Suède et en Italie, les filières $\mathrm{AB}$ sont présentées comme étant performantes. Bien organisées, elles répondent à la demande croissante des consommateurs pour des aliments exempts de traitements chimiques. Cette performance peut être exposée en quelques chiffres. En Allemagne, l'agriculture biologique représente $4,5 \%$ de la SAU, en Italie $6,2 \%$, au Danemark $5,8 \%$, en Suisse $11,3 \%$ et en Suède $6,8 \%$. Le marché bio est caractérisé comme mature en Autriche, au Danemark, en Allemagne et en Suisse, ainsi qu'au Royaume-Uni. En Finlande, en France, en Italie, aux Pays-Bas, en Norvège, au Portugal et en Suède, il est en développement. En France la ou plutôt les filières AB sont relativement peu structurées, malgré un effort récent d'organisation impulsé par une forte demande. Certaines régions sont plus particulièrement affectées par l'absence d'organisation. En Île-de-France, les producteurs ne sont pas très nombreux au regard des autres régions françaises mais le Conseil Régional incite fortement à son expansion. Afin de lire de façon intelligible les territoires du système $A B$, il convient de les effeuiller, du national au régional.

\section{L'agriculture biologique, des enjeux généraux de politique agricole}

\section{Une présentation générale du système $A B$ national}

Début 2008, 11978 exploitations agricoles étaient engagées dans la production biologique avec 557133 ha, soit $2 \%$ de la surface agricole utilisée (SAU) nationale. Ce pourcentage peut paraître faible. Toutefois, il doit être mis en comparaison avec les années précédentes. En effet, de 2001 à 2007, une progression moyenne du nombre d'exploitations bio de $2,2 \%$ par an a été enregistrée dans un contexte où le nombre total d'exploitations agricoles était en baisse chaque année (statistiques produites par l'Agence Bio en 2009 sur l'année 2008). En 1995, l'État recense 4000 exploitations agricoles certifiées $A B$, en 1999 près de 8000 , soit le double. En 2004, le nombre est en baisse par rapport l'année précédente mais la baisse ne dure qu'une année puisqu'en 2005, le nombre d'exploitations $A B$ repart en hausse et devient même supérieur à celui de 2003. Ceci s'explique en partie par un nombre important d'exploitations en conversion en 2003 qui obtiennent leur certification en 2005-2006.

7 Pour plus de deux producteurs sur trois, la totalité du chiffre d'affaire est liée à l'activité bio. Le reste du chiffre d'affaire peut être lié à une activité agricole conventionnelle ou non agricole : activité touristique (près de $11 \%$ des producteurs $A B$ ) ou activité pédagogique ( $5 \%$ des notifiés), par exemple.

8 Le système $\mathrm{AB}$ n'est pas composé uniquement de l'activité agricole. Les revendeurs, les négociants constituent une autre partie de la filière. 6402 entreprises de préparation et 
de distribution de produits $A B$ étaient certifiées en France en 2007. Les entreprises de transformation, de conservation et de conditionnement de produits $A B$ sont diversifiées, mais le secteur végétal prédomine. Enfin, l'aval de la filière se matérialise, également, par la consommation. En 2007, le marché des produits alimentaires issus de l'AB était évalué à 1,9 milliard d'euros, soit $1,2 \%$ du marché alimentaire total. Ce marché est en augmentation constante. Si la consommation de produits $\mathrm{AB}$ en France est ancienne, elle a connu depuis les années 1990 un fort développement. Le baromètre de consommation et de perception des produits « biologiques ", réalisé en octobre 2007, montre que plus de quatre Français sur dix consomment des produits « bio » au moins une fois par mois, $23 \%$ au moins une fois par semaine dont $6 \%$ tous les jours. Cet engouement pour les produits $\mathrm{AB}$ a interpellé les politiciens. Au niveau national, plusieurs décisions ont été prises.

\section{Un boom de l'AB qui interpelle et fait réagir les acteurs politiques}

Les mesures politiques se caractérisent par plusieurs types d'initiatives. La plus importante est le Grenelle de l'environnement qui se déroule de juillet à octobre 2007. L'agriculture est concernée par les mesures prises, notamment l'instauration d'une certification "Haute Valeur Environnementale ». Des objectifs très concrets sont arrêtés : un triplement des surfaces en $\mathrm{AB}$; c'est-à-dire un passage de $2 \%$ de la SAU totale à $6 \%$ en l'espace de 5 ans, une proportion de $20 \%$ de produits $A B$ dans la restauration collective d'État avant 2012.

10 Le Grenelle se voit conforté par le plan Barnier «Agriculture biologique : horizon $2012 »$ en septembre 2007. Ce plan consacre trois millions d'euros par an pendant 5 ans, pour la structuration de la filière. Il crée aussi un Comité Scientifique de l'agriculture biologique ainsi qu'un Réseau Mixte Technologique agriculture biologique constitué d'organismes de développement agricole, d'établissements de recherche, de lycées agricoles et de chambres consulaires. Ce plan est accompagné aussi du Plan National de Développement Agricole du ministère de l'Agriculture et de la Pêche qui a pour objectif de soutenir le développement économique des territoires agricoles. Cependant, il ne faut pas oublier qu'il existe deux plans précurseurs pour le développement de l'agriculture biologique (un en 1997 et un en 2002) mais tous deux n'ont pas eu d'impact significatif (Poursinoff, 2008). Ces mesures peuvent être renforcées par des politiques locales.

\section{Le système $A B$ en île-de-France}

11 L'Île-de-France a pour particularité d'être une région perçue essentiellement comme urbaine, étant donné l'emprise spatiale de la métropole-capitale. Certes, la région francilienne est urbaine puisque la première couronne a une emprise forte sur l'île-deFrance mais la moitié du territoire est en zone agricoles ou boisée et récréative. Le conseil régional exprime sa prise en compte de l'utilité d'une agriculture respectueuse de l'environnement par le biais de ses politiques environnementales. Cette collectivité locale favorise l'AB par l'attribution de subventions. Celles-ci sont justifiées tant par la protection des nappes phréatiques, que par l'approvisionnement en produit $A B$ des consommateurs parisiens. Cette collectivité territoriale parie sur le développement de l'AB. Toutefois la région reste classée avant dernière en France en nombre d'exploitations bio, 115 en 2010 (fig. 1). 
12 fonctions d'aval de la filière. Le dispositif de distribution et de vente des produits $\mathrm{AB}$ est composé de douze marchés bio, 250 enseignes bio, 40 grossistes et négociants, dont 15 sont basées au Marché d'Intérêt National de Rungis. À Sainte-Geneviève-des-Bois, dans l'Essonne, une plateforme Biocoop (la $4^{\mathrm{e}}$ plateforme créée par la société coopérative sur le territoire français) a été mise en place pour alimenter les magasins parisiens et la région "centre-nord-est » (de Lille à Strasbourg). Cette structure importante connaît régulièrement des ruptures de stock, tant la demande est en forte croissance et l'offre faible. La vente directe sous forme de paniers, dont ceux des AMAP (Association pour le Maintien d'une Agriculture Paysanne), la vente à la ferme, les marchés, occupe une place importante dans le processus de mise en marché. Le système est étayé par un petit nombre d'organismes de développement qui sont le GAB (Groupement d'Agriculture Biologique) Île-de-France, les chambres d'Agriculture (Seine-et-Marne, chambre inter-départementale Île-de-France), l'Établissement Régional de l'Élevage, le duo INRA-AgroParisTech, les lycées agricoles (à Brie-Comte-Robert lycée de Bougainville, à Chailly-en-Brie lycée de la Bretonnière dans la Seine-et-Marne et celui de la Bergerie Nationale à Rambouillet dans les Yvelines), ainsi que certains établissements privés (Maisons familiales rurales, lycées). Ils contribuent à la mise au point et à la diffusion de ressources technologiques.

Figure 1 : Localisation des agriculteurs bio au sein du contexte francilien/Localisation of organic farmers in Paris Region

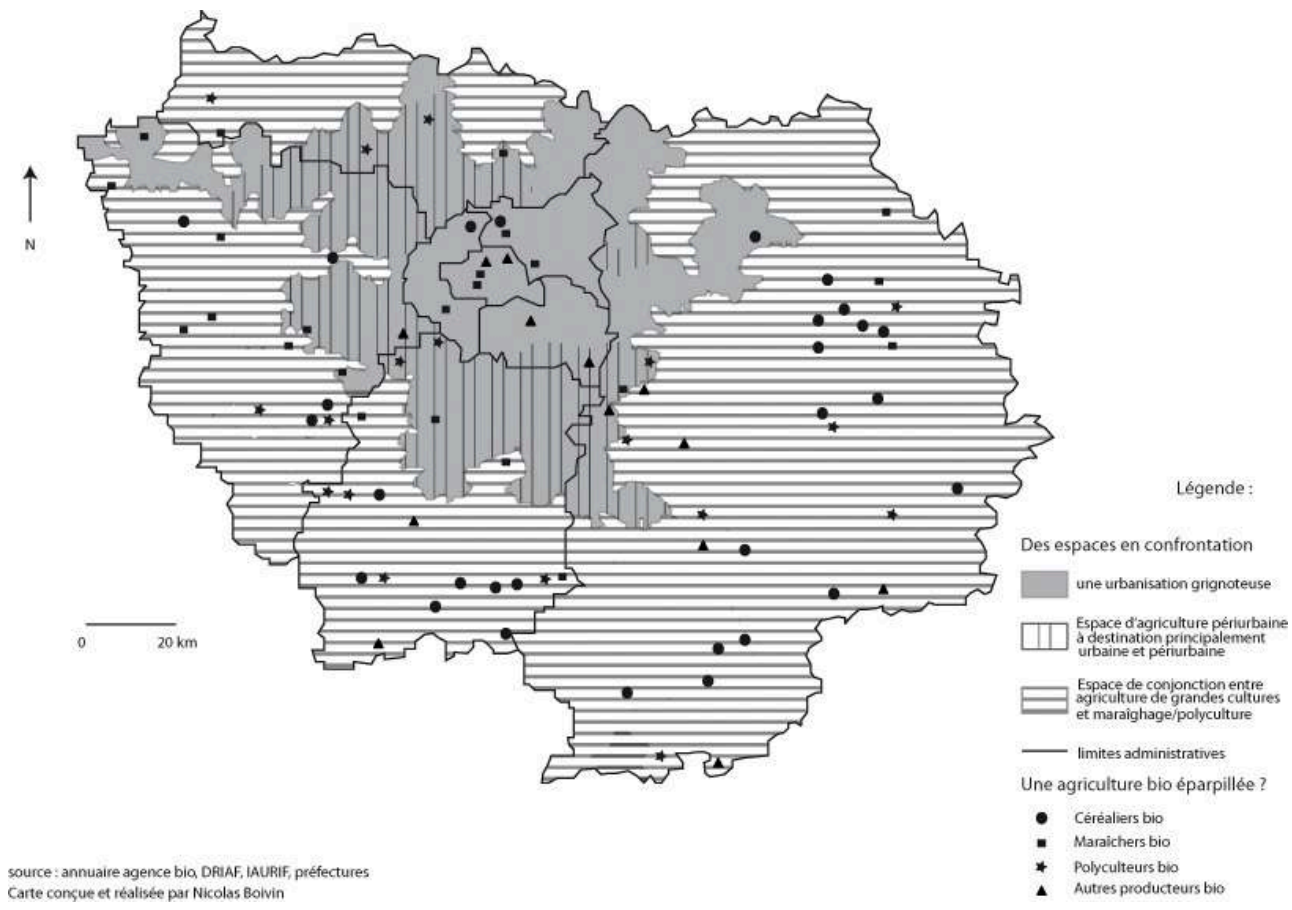

Le Conseil Régional en appelle aux prescriptions de l'AB pour résoudre certaines problématiques qui lui sont posées. Ce sont d'une part, les exigences de prise en compte des questions techniques précises, pollution de l'eau, renouvellement d'une population agricole vieillissante, aménagement de l'espace que l'agriculture conventionnelle échoue à résoudre. Pour cela, cette autorité souhaite l'instauration d'un système $A B$ doté d'une gouvernance propre alliant tous les acteurs présents et futurs de l'AB. L'invocation, quasi incantatoire du bio, est aussi affichée comme la solution alternative 
indispensable pour répondre aux inflexions annoncées de la PAC en 2013. L'ensemble de ces acteurs fabrique du territoire alternatif au monde conventionnel. Il interagit en faveur de modifications marginales mais visibles. Pour comprendre ces changements, nous envisageons d'abord d'exposer les mécanismes qui forment la base des possibles territorialisations alternatives.

\section{Les rouages actoriels du système $A B$ en Île-de-France ou comment se joue la construction d'une alternative?}

14 L'« agriculture biologique » forme-t-elle un système territorial alternatif ? Quelle est véritablement la nature et la portée du schisme qui se produit dans la région parisienne en terme d'agriculture ? L'AB porte-t-elle une véritable alternative dans le contexte spatial de l'île-de-France si l'on accorde un double sens à ce concept ? Pour répondre à cette question, l'analyse des stratégies et des comportements des acteurs des différents domaines $\mathrm{du}$ jeu que sont les domaines économique, politique, social et organisationnel, est indispensable. Une première lecture met en évidence des interactions fortes entre ces quatre domaines, qui ne définissent pas pour autant un système territorial. Les difficultés d'intégration des produits $A B$ dans les circuits économiques conventionnels sont révélateurs des difficultés d'existence du système $A B$ d'Île-de-France. Elles ne présument pas pour autant du caractère alternatif de cette forme de production et de sa capacité à renouvellement les formes territoriales. En agissant les acteurs mettent en place un système à la marge de l'agriculture conventionnelle. Toujours en première analyse, c'est encore cette dernière qui caractérise pour le moment, la production agricole francilienne et qui tient les principaux leviers d'action économique. La vision manichéenne de l'agriculture contemporaine que les acteurs, agriculteurs et non agriculteurs, se plaisent à présenter ne peut suffire comme élément explicatif de la trajectoire de développement contemporaine de l'agriculture et des territoires de l'île-de-France.

La région Île-de-France comporte effectivement deux modèles différents d'agriculture. Un premier modèle basé une agriculture productiviste et intensive en mécanisation et en intrants chimiques et la commercialisation de ses produits par la grande distribution, et un second modèle en marge qui se positionne principalement sur la consommation locale et les circuits courts suivant le schéma classique d'organisation productive des systèmes agricoles alternatifs (Aubry et Chiffoleau, 2009). L'agriculture biologique utilise les deux systèmes. Cette agriculture est, également, alternative au point de vue spatial puisqu'elle se retrouve enclavée entre les grandes cultures et l'urbanisation "grignoteuse ». Elle est tiraillée par un dualisme incessant, entre une insertion dans le monde « conventionnel » et une possibilité de création d'alternative.

\section{L'organisation francilienne de la vente des produits issus de l'agriculture biologique, la fabrique d'un système alternatif ?}

Les enquêtes ${ }^{3}$ montrent que les agriculteurs utilisent une pluralité de circuits de commercialisation. Fait essentiel, ils privilégient les circuits courts, ce n'est pas très surprenant d'autant plus que ces circuits sont " promotionnés » par les organismes qui s'occupent de l'agriculture biologique. Ces mises en marché locales s'observent chez les maraîchers qui vendent soit directement aux consommateurs qui se rendent sur 
l'exploitation, soit sur les marchés de plein vent ${ }^{4}$. Ce choix commercial est motivé par deux raisons. Tout d'abord, les produits frais gardent leurs qualités en étant commercialisés rapidement. Deuxièmement, l'agriculteur capte une marge commerciale qui lui permet d'accroitre un revenu agricole généralement faible dans les exploitations maraichères, d'autant plus qu'en $\mathrm{AB}$, les exploitations sont de petite taille et privilégient des rendements bas. Les maraîchers et les producteurs d'herbes aromatiques sont souvent liés à des associations de consommateurs qu'ils fournissent en paniers (Lamine, 2008). Compte tenu de la forte demande en paniers, ils privilégient de plus en plus des associations locales. Les maraîchers et les producteurs de légumes en plein champ interviewés alimentent des associations de plus en plus proches, dans des rayons de moins de $20 \mathrm{~km}^{5}$. Ils refusent aujourd'hui les demandes d'associations parisiennes pour éviter les problèmes de circulation et s'investissent davantage auprès d'AMAP rurales ou périurbaines plus récentes que les AMAP du centre de Paris. Les céréaliers vendent à des coopératives conventionnelles qui possèdent un ou deux silos réservés aux céréales certifiées $A B$, distants en majorité de moins de $30 \mathrm{~km}$. Le débouché coopératif est rendu possible grâce au maillage serré des coopératives céréalières dans le bassin francilien.

Toutefois, les producteurs utilisent aussi des circuits de moyenne distance. Ils approvisionnent directement les Biocoop qui couvrent une zone de chalandise de l'ordre du département, un maillage administratif médian en France. La superposition des mailles de distribution fonctionne par le biais des interrelations territoriales. Il s'agit pour les acteurs de se rencontrer et de réaliser des transactions satisfaisantes aussi bien pour l'acheteur que pour le consommateur. En effet, les retours d'expérience des interrogés montrent que les consommateurs attendent des produits bio une proximité, ils veulent consommer local pour soutenir l'économie locale ainsi que pour connaître la provenance exacte des produits. De ce choix et de ces réseaux naissent des nœuds, des noyaux fonctionnels. Les marchés, quelques soit leur nature, sont des " hubs ». Ils ont une fonction logistique et redistributive qui permet l'orientation des produits vers la demande finale. Ce sont les lieux d'échanges et d'interactions entre différents types d'acteurs afin de satisfaire les attentes territoriales. L'espace francilien connait trois plaques tournantes pour la bio.

- Tout d'abord, la capitale, elle-même, est le premier hub. Une aire urbaine de cette importance nécessite un « échangeur alimentaire » où se constituent des réseaux alternatifs d'achalandage (Jarosz, 2008).

- Le deuxième pôle est la plateforme logistique de Biocoop installée récemment en banlieue parisienne. Biocoop, premier réseau de magasins bio en France, a réalisé 250 millions d'euros de chiffre d'affaires en 2006, soit une progression de $22 \%$ par rapport à 2005 . Ce rythme de croissance dépasse celui du marché des produits alimentaires bio qui, d'après les récents chiffres de l'Agence Bio, a enregistré une croissance moyenne de 9,5\% depuis 1999. Biocoop possède $12 \%$ des parts de marché des produits biologiques français. En cinq ans, 100 points de vente supplémentaires Biocoop ont ouvert, portant à 274 le nombre total de magasins. Le quart Nord-Est de la France est la zone vouée au plus fort développement. Pour anticiper cette progression, une nouvelle plateforme située à Sainte-Geneviève-des-Bois, en région parisienne a ouvert ses portes le 16 janvier 2007. Ce nouvel équipement rapproche un peu plus Biocoop de son objectif de proximité. Elle dessert les 42 magasins du quart Nord-Est de la France, qui étaient auparavant approvisionnés par la plateforme de Rennes. Ce hub a pour caractéristiques techniques $7732 \mathrm{~m}^{2}$ dont $1200 \mathrm{~m}^{2}$ de froid, $400 \mathrm{~m}^{2}$ de mixage, une capacité de 6000 palettes, un choix de 3420 produits pour le secteur sec alimentaire, 1380 
produits pour le secteur sec non-alimentaire, 800 produits ultra-frais, 130 fruits et légumes. Ce nœud bio a permis la création de 61 emplois.

- Le troisième hub est le Marché d'Intérêt National de Rungis. Ce marché s'étend sur 600 ha, reçoit plus de 500 wagons par jour et cumule $17 \mathrm{~km}$ de quais pour desservir les $150000 \mathrm{~m}^{2} \mathrm{de}$ stand. Ce MIN a un rayonnement national et international. Si les produits $A B$ y sont largement minoritaires il est à noter qu'il y a en permanence trois négociants bio et que les maraîchers bio peuvent déposer leurs produits au Carreau des Maraîchers. Tous les maraîchers interrogés livrent une part de leur production à Rungis, soit plusieurs fois par semaine, soit épisodiquement, pour écouler des excédents. Les producteurs de plantes aromatiques $^{6} \mathrm{y}$ trouvent un débouché très lucratif.

18 Les acteurs économiques, productifs et commerciaux, ne participent pas seuls à la structuration du système. Ils sont accompagnés par des acteurs politiques qui régulent et soutiennent la bio, en particulier le Conseil Régional. Des interventions politiques complètent la sphère des interactions qui participent de la création d'un système alternatif. L'action des uns apporte à l'action des autres.

\section{Des acteurs politiques en chemin vers une économie régionale alternative?}

19 La Région Île-de-France veut mettre en place, depuis 2007, la première Ecorégion d'Europe. Dans cette optique elle favorise le développement de l'agriculture biologique à partir de trois groupes d'actions programmées pour la période 2009/2013 :

- Le Programme d'Actions Régional Concerté pour l'Agriculture Biologique (PARC BIO) qui permet d'intervenir directement en faveur du développement de la filière bio en IDF. Ce programme permet à la Région d'exercer un contrôle partiel du foncier en travaillant avec les SAFER, d'apporter des solutions aux agriculteurs en finançant le GAB et d'attribuer une prime d'installation, etc.

- L'Aide Régionale au Maintien de l'Agriculture Biologique (ARMAB) pour soutenir les producteurs qui sont déjà convertis afin qu'ils puissent bénéficier du meilleur encadrement technique et financier, l'ARMAB se substitue aux CTE.

- L'introduction des produits bio dans la restauration collective.

Le plan de développement de l'agriculture biologique voté en juin 2009 est issu d'un processus de démocratie participative organisé par une élue verte au cours de plusieurs ateliers consacrés à la bio et une pluralité de profils de participants. Cette consultation a permis de réunir environ 450 acteurs de la bio et de l'agriculture conventionnelle. Cette démarche doit permettre l'installation et la conversion en $A B$.

21 Avec ce plan d'action, la Région rompt avec un schéma d'intervention en faveur de la seule agriculture conventionnelle. Elle promeut une économie alternative au système dominant. Le plan de la région se démarque de la stratégie des principales organisations d'agriculteurs historiquement opposées à l'AB comme la FNSEA. Plusieurs innovations sont mises en place. La Région intervient auprès de la SAFER pour identifier et délimiter des périmètres destinés préférentiellement à l'installation en $\mathrm{AB}$. Elle finance des projets d'économie sociale avec l'association Terre-de-liens. Elle soutient des initiatives collectives pour l'acquisition de foncier et la création de " couveuses agricoles». Ces dernières sont des fermes expérimentales destinées à l'installation agricole de porteurs de projets hors cadre d'installation familiale. Les Centres d'Initiatives pour Valoriser l'Agriculture et le Milieu Rural (CIVAM) souhaitent 
démontrer que l'installation progressive est possible dans le cadre du Système Alimentaire Local (vente de proximité, AMAP...). Les couveuses proposent des «espaces-tests » pour de futurs entrepreneurs: les couvés. C'est l'apprentissage du métier d'agriculteur et la confrontation à la réalité qui valideront les projets des couvés. La couveuse met à disposition des moyens de production, un accompagnement humain, technique, financier et administratif. La Région intervient aussi pour compenser la spéculation foncière qui a lieu dans les espaces périurbains en participant au financement de foncier rendu à sa destination agricole.

Cet ensemble d'actions et d'acteurs permet d'envisager l'AB francilienne comme une trajectoire alternative potentielle. Il autorise la mise en place d'une gouvernance alliant acteurs individuels, acteurs collectifs, acteurs de la production, acteurs civils, acteurs associatifs, acteurs institutionnels et acteurs politiques, un mode de changement qui se base sur des territorialisations de proximité.

\section{Vers un système $A B$ vraiment alternatif ?}

23 Nous avons vu que le premier acteur politique régional souhaite instaurer une nouvelle dynamique de développement de l'agriculture sur une base AB. Ce projet est constitutif d'un projet plus vaste, la création de la première Ecorégion d'Europe. Cette programmatique régionale impacte directement l'aménagement du territoire. Une nouvelle territorialisation voit le jour en s'appuyant sur une culture du développement économique qui ne s'inscrit pas dans le schéma dominant. Ces caractères suffisent-ils à qualifier ces territoires en formation d'alternatifs? Ou créent-ils une variante territoriale au sein de cette Région particulière?

\section{La transfiguration du système francilien... des espaces bio qui se complexifient}

24 Les espaces ruraux franciliens apparaissent comme complexes même s'ils ont pour dénominateur commun d'être sous influence d'une importante agglomération urbaine (Poulot et Rouyres, 2007 ; Bryant, 1997 ; Donadieu et Fleury, 2003). Ces espaces agricoles se sont transformés afin de permettre une agriculture extensive et productiviste. Dans le même temps, ils ont du supporter en leur propre sein une urbanisation, synonyme d'accueil d'usagers aux exigences fortes. Ces exigences diffèrent de celles des habitants historiques des espaces ruraux en termes d'équipements, d'environnement et d'aménagement paysager (Poulot et Rouyres, 2007). Pour décrire les changements engendrés par l'agriculture biologique, nous proposons tout d'abord de présenter le cadre général des espaces productifs des campagnes franciliennes. Ces espaces agricoles sont découpés en deux parties inégales, une ceinture maraîchère et fruitière, mise à mal par l'essor des infrastructures de transports et des espaces céréaliers où de grosses fermes aux gigantesques parcelles et avec très peu de main d'œuvre s'intercalent entre les bourgs des plateaux (ibid.). Selon ces auteures, la vente à la ferme est commune chez les maraîchers et les producteurs d'œufs. Environ 30 exploitations ont créé des activités de cueillette afin de répondre à la demande urbaine. La tendance est à l'agriculture de proximité portée par différents types de labels, marque Parc naturels, labels rouges, AOC. L'agriculture biologique s'inscrit parfaitement dans ce 
mouvement alternatif de développement des circuits courts et de l'agriculture dite urbaine (Aubry et Chiffoleau, 2009).

En accueillant de nouveaux occupants et de nouvelles activités les campagnes franciliennes sont devenues multifonctionnelles. Elles apparaissent de plus en plus comme des espaces de production, mais aussi et surtout en tant que cadre de vie des citadins (Poulot et Rouyres, 2007). Lors des enquêtes que nous avons menées, les agriculteurs bio évoquent le fait que leur installation ou leur conversion est perçue comme une intrusion dans le monde de l'agriculture conventionnelle. Au départ, ces agriculteurs sont peu acceptés par leurs voisins agriculteurs. Leur intégration n'est possible que sur la preuve de la pérennité de leur système d'exploitation. Certains agriculteurs bio parviennent même à obtenir une reconnaissance de leur compétence technique. Le regard porté par les populations non-agricoles est différent. L'AB est assimilée à une pratique bénéfique pour la santé et l'environnement. Elle jouit à ce titre d'un a priori positif. Nous qualifions cette perception par l'acronyme YIMBY. Cette perception fait contrepoids aux manifestations NIMBY. Le " yes » remplace le « no ». L'agriculture des "campagnes urbaines » se fond dans un environnement aménagé pour des populations citadines qui bannissent les éléments de l'agriculture moderne. Le développement des pratiques «environnementalement respectueuses" des agriculteurs périurbains récemment installés s'accorde parfaitement avec les logiques citadines de reconstitution d'un Éden naturel! (Donadieu et Fleury, 2003). L'AB offre une solution alternative à celle de la sphère agricole francilienne. Elle traduit concrètement les aspirations de populations de culture citadine, qu'elles vivent dans des espaces urbains, périurbains ou ruraux.

Afin d'apprécier plus finement la transition qui s'opère dans le système francilien, nous la comparons à celle analysée par Mendras lors de l'introduction du maïs hybride dans le Sud Ouest à partir des années 1950 (Mendras, 1984). Bien qu'anciens, ces travaux sont pertinents pour comprendre les mutations d'un système agricole sous l'effet de l'introduction d'une alternative technologique. Malgré un rendement supérieur du maïs hybride constaté par les agriculteurs des Basses Pyrénées, les producteurs béarnais ont continué à semer deux sortes de maïs, de l'hybride et du Maïs de pays. Les agriculteurs rechignaient à changer totalement de système de production pour différentes raisons, autant techniques et économiques que culturelles. Ils adopteront une voie intermédiaire au tout moderne en introduisant progressivement les innovations variétales et mécaniques. Dans le cas de l'AB, les objectifs du changement technique diffèrent. Ils sont beaucoup plus radicaux. Il s'agit d'une sortie du paradigme sur lequel a reposé le développement agricole en France. L'AB propose de réduire l'utilisation d'intrants, de limiter les rendements et d'augmenter la charge de travail. C'est l'exact contraire des objectifs assignés à l'agriculture francilienne depuis soixante-dix ans. Deux stratégies de conversion en AB co-existent. Les conversions partielles sont motivées par des capacités d'investissement trop réduites. Durant les trois années de la phase de conversion, les rendements de l'exploitation baissent sans que le producteur bénéficie du label $A B$ et des prix correspondants. Ce handicap, compensé pendant un temps par les Contrats Territoriaux d'Exploitation ne l'est plus depuis que ceux-ci ont été remplacés par les Contrats d'Agriculture Durable (CAD). La seconde option, plus radicale, est de convertir entièrement l'exploitation. Les agriculteurs rencontrés qui ont choisi cette solution sont généralement les plus motivés par une éthique environnementale (Van Dam, 2007). 
28 Le maïs hybride a été l'avant-garde d'une série de modifications du système agronomique du sud-ouest. Au plan technique, la conduite de la parcelle diffère par rapport à la culture des variétés locales. Le maïs hybride beaucoup plus exigeant en intrants a contraint les agriculteurs à s'endetter. Il ne faut pas oublier non plus que ce maïs a fait entrer sur l'exploitation le conseiller agricole. Concomitamment l'augmentation des surfaces en maïs fourrager a permis un changement de spécialisation des éleveurs, ceux-ci passant de la production de broutards à la production de lait un temps plus rémunératrice et lourdement subventionnée. Pour l'agriculture bio en Île-de-France, les processus du changement sont similaires même si les objectifs sont très différents. L'agriculture biologique requière un changement de technologies qui semblent la rendre plus technique que l'agriculture conventionnelle. Les conseillers agricoles des chambres d'agriculture et des coopératives, du GAB, les consultants privés deviennent des interlocuteurs incontournables pour les producteurs qui n'ont pas eu de formation sur les pratiques de l'agriculture bio, en particulier les agriculteurs en conversion. L'agriculture bio demande aussi une diversification des cultures à cause des rotations obligatoires pour le maintien de la fertilité et de la structure des sols. A ces contraintes agronomiques nouvelles pour les agriculteurs du $\mathrm{xxI}^{\mathrm{e}}$ siècle s'ajoute la diversification des activités productives qui augmentent la complexité de la gestion de l'exploitation bio. La vente en circuits courts permet d'écouler plus facilement ces divers produits notamment par le biais des ventes à la ferme ou sous forme de paniers qui imposent une gestion logistique lourde.

À propos de la conversion au maïs hybride, Mendras rappelle que le processus a été long. Les pionniers fournissent un ou des exemples attentivement observés par les agriculteurs voisins. La diffusion se fait de façon progressive. Il semble en être de même pour les pratiques de l'AB. Cette forme d'agriculture ne modifie pas que les rapports de l'agriculteur à ses pratiques. Elle change son rapport à la terre, aux formes d'occupation $\mathrm{du}$ foncier. Elle touche même largement son rapport au territoire aux échelles locale et régionale. De nouvelles zones d'influence apparaissent sur la carte francilienne.

\section{Alternative et échelons local/régional : des territoires nouveaux ?}

L'agriculture biologique n'est pas d'essence locale. Cependant, les consommateurs et les acteurs politiques souhaitent structurer cette agriculture à une échelle régionale. Les enquêtes auprès d'agriculteurs bio nous ont permis de mesurer précisément la forme et les distances de leurs réseaux. Ils apparaissent nettement attachés à des réseaux de proximité. Ces relations au proche semblent même plus développées en $A B$ qu'en conventionnel. Les agriculteurs échangent localement et régionalement. Ces interactions avec le proche concernent principalement les ressources mobilisées par les agriculteurs. Nous avons pu recenser une fréquence élevée de distances d'environ $15 \mathrm{~km}$ qui témoignent de cette relation forte au proche.

Il ressort également que la terre reste une ressource fondamentalement ancrée à un espace proche. Les maraîchers expriment le plus clairement les difficultés d'accès à la terre. Pourtant leurs besoins se limitent à de petites structures, qui peuvent s'encastrer plus facilement entre les aires accaparées par une urbanisation grignoteuse (fig. 1). Les polyculteurs, un peu plus excentrés, ne connaissent pas spécialement de pression, étant donné qu'ils convertissent des anciennes parcelles céréalières en «espaces 
maraîchers ». Les terres agricoles sont situées dans un rayon de moins de $13 \mathrm{~km}$ de leur exploitation.

Le matériel végétal, semences et plants, est la seconde ressource matérielle importante. Il peut être local, régional ou d'origine plus lointaine. Les agriculteurs peuvent produire eux-mêmes, graines et plantes certifiés $\mathrm{AB}$, mais c'est techniquement contraignant. La fourniture en graines et plants se fait par plusieurs voies. En premier lieu les agriculteurs procèdent par échanges. Certains exploitants du sud de la Région vont même jusqu'à se spécialiser dans la production de semences et plants $A B$. Les coopératives de céréales jouent également un rôle important dans la fourniture de graines $\mathrm{AB}$. Ce réseau privilégie également une logique de proximité géographique. Mais il faut relever que la fourniture de ces intrants $A B$ se produit essentiellement à une échelle supérieure, sur la base de flux distants. Une particularité de l'île-de-France, les producteurs de plantes aromatiques, un marché important notamment pour ceux qui livrent à Rungis ou à des GMS spécialisée, ont la possibilité d'acheter plants et graines au Conservatoire National des Plantes Aromatiques situé à Milly-la-Forêt dans le sud de l'Essonne. Un processus de territorialisation est en phase d'amorçage. Les maraîchers du sud de l'Essonne et du sud de la Seine-et-Marne produisent des plantes aromatiques $\mathrm{AB}$ incités par la conjonction d'un marché rémunérateur, Rungis et les magasins bio, de coûts de transport faibles, du fait de la proximité spatiale, et la facilité de l'approvisionnement en graines et plants.

Au final les exploitants échangent des quantités importantes de matériels et de savoirfaire. Ces échanges sont soit spontanés, au cours de rencontres informelles entre agriculteurs proches, soit organisés par les organisations du développement agricole. Ces flux matériels et immatériels ne se limitent pas au niveau régional. Nonobstant, ces «trocs» régionaux paraissent plus variés et denses en $\mathrm{AB}$ qu'en agriculture conventionnelle.

La vente locale ou régionale est fortement valorisée par les producteurs rencontrés. À partir des flux commerciaux individuels nous avons élaboré une carte en guise de première représentation des territoires proches des agriculteurs bio franciliens (fig. 2).

Cette carte est une ébauche et ne doit pas être abordée comme une carte respectant la sémiologie graphique habituelle en géographie. Elle est issue d'une base déclarative constituée lors des entretiens avec le quart de la population d'agriculteurs bio de la région. Elle n'est donc pas exhaustive, mais c'est un échantillon suffisamment conséquent pour transcrire la réalité des pratiques de mobilités. Le territoire quotidien du métier d'agriculteur bio est compris dans un rayon de $15 \mathrm{~km}$ autour du siège d'exploitation. Le concept de cette carte aussi simple qu'il soit, permet de matérialiser l'espace de relations de chaque producteur bio. Une transparence de $60 \%$ permet de superposer les cercles (fig. 2) et de représenter à partir de zones plus foncées les territoires qui s'inscrivent plus intensément dans une alternative au modèle dominant (production, inscription dans le local et le régional). Après confrontation de la délimitation de ces aires à d'autres données, nous avons élaboré une seconde carte en intégrant le découpage communal. Cette troisième carte (fig. 3) représente donc les zones d'influence les plus marquées, les territoires dont nous faisons l'hypothèse qu'ils exercent la plus forte influence en faveur d'une dynamique alternative dans la Région.

La figure 3 représente les territoires où l'AB est une alternative consistante à l'agriculture conventionnelle. Ces territoires sont complétés par des territoires où la bio est en voie de devenir une possible alternative. Des fronts pionniers sont 
identifiables. Nous remarquons que les Yvelines sont un territoire alternatif conséquent (en devenir et de fait). Ceci s'explique par la prégnance des politiques de préservation des espaces et des paysages dans ce département. Au sud de l'Essonne, un véritable pôle associant maraichage et céréaliculture se démarque. Il est né de l'initiative de producteurs leaders qui parviennent à entrainer d'autres producteurs en les convainquant de profiter des opportunités offertes par le marché de Rungis. Il faut souligner la présence d'autres hubs moins connus comme la plateforme biocoop de Sainte-Geneviève-des-Bois et les magasins spécialisés Naturéo (filiale d'Intermarché). Pour finir, deux zones sont visibles en Seine-et-Marne. Celle de l'ouest du département tire bénéfice de la capitale et de son marché urbain qui offre un débouché aux systèmes de commercialisation en paniers et à des produits comme le miel ou le cidre. Celle du centre est due essentiellement au développement d'une filière lin. À la recherche de débouchés, les céréaliers investissent dans cette culture destinée à l'industrie textile et à l'industrie du bâtiment qui l'emploi comme isolant. Par un effet d'entrainement ces mêmes céréaliers se convertissent peu à peu à la production de légumes plein champ afin de compléter leur revenu. Paris intramuros a été évincé volontairement de la représentation de ces territoires alternatifs. C'est un cas particulier qui devra faire l'objet d'une analyse de processus qui, s'ils ne sont pas spécifiquement urbains, sont toutefois très marqués par le contexte parisien (jardins familiaux, AMAP, boboïsation de l'habitat et de la gestion des infrastructures publiques...).

Figure 2 : Pôles et échanges de proximité des producteurs bio/Spaces mobilities of organic farmers

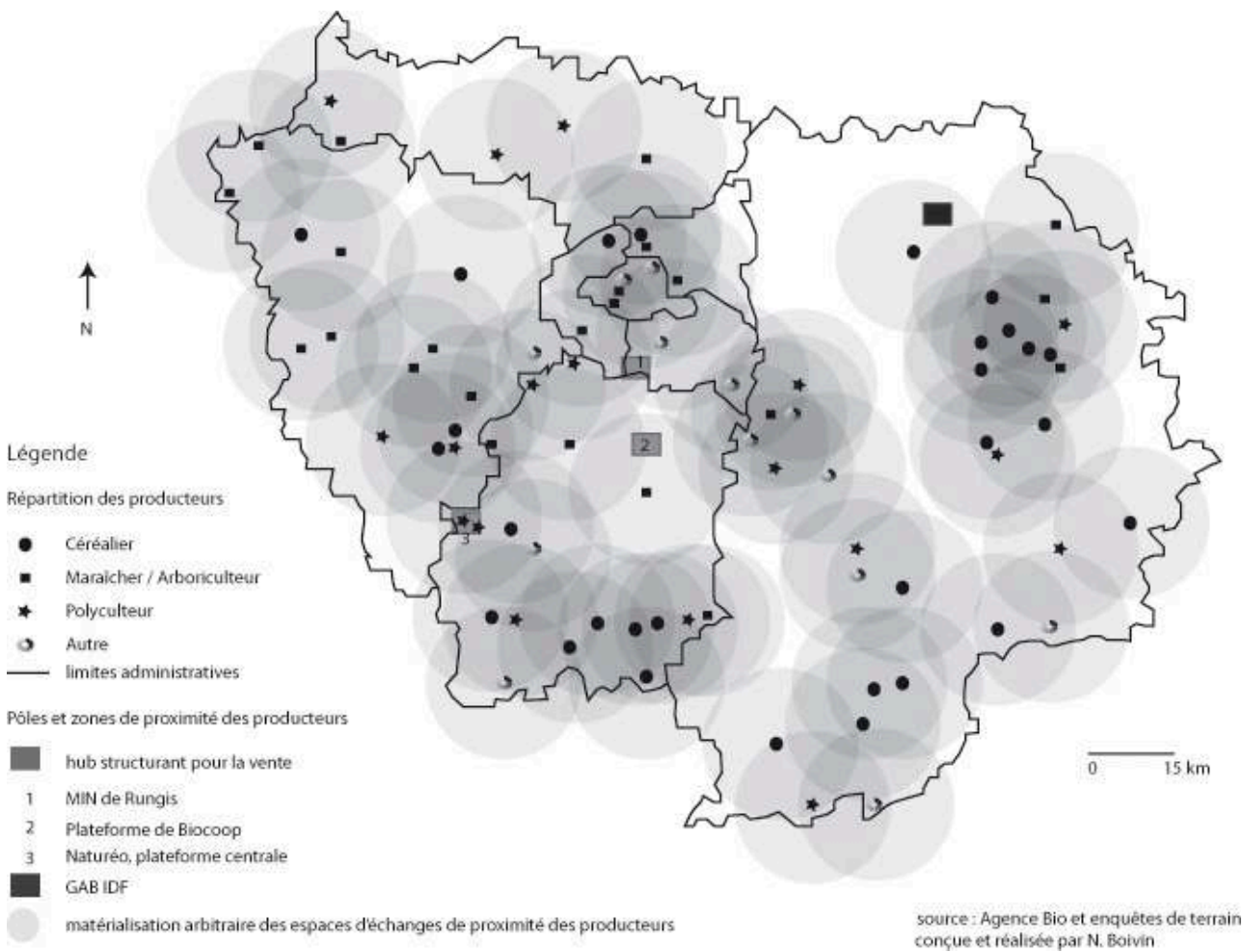

Les zones qui se dirigent vers une territorialisation possible à partir de l'AB sont, soit à la périphérie des précédentes dans une logique de conquête spatiale, soit dans des espaces où les acteurs misent sur la préservation de leur cadre de vie et préfèrent une agriculture YIMBY à une agriculture productiviste. C'est le cas des territoires en périphérie de la forêt de Fontainebleau. 


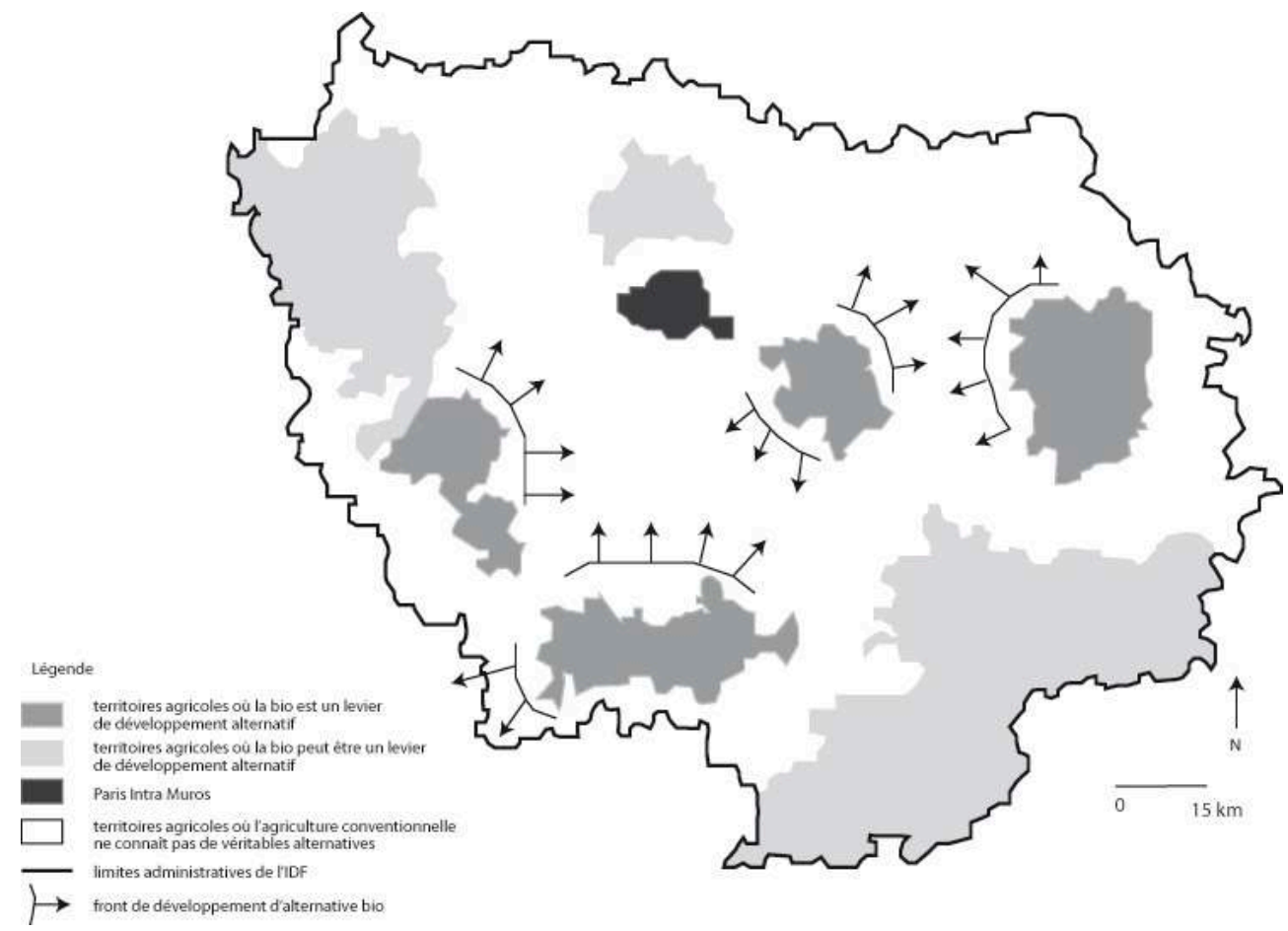

\section{Conclusion}

L'agriculture biologique est une activité marginale des espaces périurbains et ruraux de l'Île-de-France. Toutefois elle offre une solution pratique et peu coûteuse, tant à des problèmes de nature technique que politique. L'excellente perception de l'AB par le consommateur/électeur en fait un argument de politique locale séduisant. En réponse à des revendications sociales qui font de plus en plus état de préoccupations sanitaires liées aux pollutions d'origine agricole ou industrielle et à l'alimentation, les acteurs politiques inscrivent ce type d'agriculture dans le cahier des charges de l'aménagement du territoire francilien. En Île-de-France l'agriculture biologique est un pilier de la mise en place de l'Ecorégion. Elle occupe dans la politique régionale une place déconnectée des indicateurs de performances admis en termes de politique économique traditionnelle. Le nombre d'emplois et d'entreprises créés est faible pour une région qui accueille près de $20 \%$ du PIB national. C'est d'abord et avant tout un atout politique et électoral accordé en contrepartie d'alliances politiques locales avec le mouvement écologiste.

Pour autant l'AB n'est pas dépourvue de vertus, que les acteurs régionaux ambitionnent d'exploiter. Pour faire face au retard de développement de cette agriculture par rapport à ce que l'on observe dans d'autres régions d'Europe, l'île-de-France met en avant son intérêt pour réduire la pollution des nappes phréatiques, les phénomènes de minéralisation des sols et le déficit de la production légumière et fruitière pour l'alimentation des populations locales. Ce système de production agricole produit des externalités environnementales mais aussi sociales et économiques. Il est consubstantiellement favorable à la diversification des productions et se présente donc 
comme une alternative au modèle productiviste en monoculture qui structure actuellement le secteur et l'espace agricole de la région.

L'agriculture biologique francilienne se construit autour de réseaux politiques, administratifs et productifs. Le développement de son potentiel de production associe l'émergence de hubs logistiques, tels que la plateforme Biocoop de Sainte-Genvièvedes-Bois ou bien sûr Rungis. Un certain nombre d'actions initiées par le Conseil Régional (galette bio, carreau des maraîchers au MIN, etc.) offrent des débouchés intéressants aux producteurs et encouragent les conversions. Cet encouragement au développement de la filière par la structuration d'une demande spécifique inscrit l'agriculture biologique dans une dynamique alternative. Les outils financiers mobilisés par les puissances publiques tout comme ses relais citoyens, administratifs et économiques sont parallèles à ceux qui soutiennent l'agriculture conventionnelle.

L'AB, de ce fait, devient une ressource transcendante qui fabrique des territoires alternatifs au système dominant. Le cœur de la Seine-et-Marne, le sud essonnien et les Yvelines dans leur plus grande partie sont des espaces où l'AB sans être majoritaire ouvre des nouvelles perspectives pour penser l'agriculture périurbaine francilienne. Ces aires accueillent un ensemble de processus gouvernanciels susceptibles de déstabiliser le régime de gouvernance conventionnel dominant et de modifier en profondeur les trajectoires de développement des territoires agricoles franciliens.

\section{BIBLIOGRAPHIE}

ALlAire G., 2002. L'économie de la qualité, en ses secteurs, ses territoires et ses mythes, Géographie, Économie, Société, $\mathrm{n}^{\circ}$ 4, p. 155-180.

Aubry C., Chiffoleau Y., 2009. Le développement des circuits courts et l'agriculture périurbaine : histoire, évolution en cours et questions actuelles, Innovations Agronomiques, n 5, p. 53-67.

BACCONNIER-BAYLET S., VIANEY G., 2006. L'aménagement communal périurbain : maintenir l'agriculture pour préserver quelle ruralité ?, Revue d'Economie Régionale et Urbaine, $\mathrm{n}^{\circ} 3$, p. 355-372.

Benasayag M., Sztulwarkal D., 2000. Du contre-pouvoir, Paris, La Découverte, 327 p.

BENOÎT J.-P., BENoîT P. et Pucci D., 2002. La France à 20 minutes. La révolution de la proximité, Belin, Paris, $271 \mathrm{p}$.

Bermond M., Croix N., Madeline Ph., Margetic Ch., Peltier Ch. et Pierre G., 2008. Durabilité, agricultures et territoires : quels questionnements pour les ruralistes d'universités de l'ouest?, Géocarrefour Agricultures, durabilité et territoires, vol. 83, n³ 3, p. 245-249.

Boschma R., 2005. Role of proximity in intercation and performance : conceptual and empirical challenges, Regional Studies, vol. 30, n 1, p. 41-45.

BRYANT C.-R., 1997. L'agriculture périurbaine : l'économie politique d'un espace innovateur, Cahiers d'Agriculture, $\mathrm{n}^{\circ}$ 6, p. 125-130. 
Clarke N, Cloke P, BARnetT C et MALPASS A., 2008. The Spaces and Ethics of Organic Food, Journal of Rural Studies, vol. 24, p. 219-230.

Colletis-Wahl K. et Pecqueur B., 2001. Territories, Development and Specific Resources : What analytical Framework?, Regional Studies, vol. 35, n 5, p. 449-459.

Delfosse C., 2008. Editorial ; agricultures, durabilité et territoire, Géocarrefour Agricultures, durabilité et territoire, vol. 83, n 3, p. 167-170.

DEVERRE C., LAMINE C, 2010. Les systèmes agro-alimentaires alternatifs, une revue des travaux anglophones en sciences sociales, Économie Rurale, n 317, p. 57-73.

Di Meo G., 1991. L’Homme, la Société, l’Espace, Paris, Anthropos, coll. « Économica », p. 144.

DiXON J., 1999. A Cultural Economy Model for Studying Foods Systems, Agriculture and Human Values, vol. 16, n 2, p. 151-160.

De LIND L. B., 2002. Place, work and civic agriculture : common fields for cultivation, Agriculture and Human value, vol $19, \mathrm{n}^{\circ} 3$, p. 217-224.

DONADiEU P. et FleUry A., 2003. La construction contemporaine de la ville-campagne en Europe, Revue de géographie alpine, vol. 91, n 4, p. 19-29.

DubuisSON-QUeLliER S., 2008. La consommation engagée, Paris, Presses de Sciences Po, 144 p.

FRIEDLAND H. W., 2001. Reprise on commodity systems methodology, International Journal of Sociology of Agriculture and Food, vol. 9, $\mathrm{n}^{\circ}$ 1, 82-104.

Goodman D., 1999. Agro-Food Studies in the "Age of Ecology" : Nature, Corporeality, Bio-Politics, European Society For Rural Sociology, Sociology Ruralis, vol. 42, n 1, p. 5-22.

HigGINS V., DiBBEN J. et CoCKLIN C., 2008. Building alternative agri-food networks : Certification, embeddedness and agri-environmental governance, Journal of Rural Studies, vol. 24, p. 15-27.

HINRICHS C., 2003. The practice and politics of food system localization, Journal of Rural Sociology, vol. $19, \mathrm{n}^{\circ} 1$, p. 33-45.

HoCHEDEZ C., 2008. Le bonheur est dans le panier. Réseaux alimentaires alternatifs et commercialisation des produits issus de l'agriculture biologique : l'exemple suédois, Géocarrefour Agricultures, durabilité et territoires, vol. 83, n 3, p. 225-233.

JAROSZ L., 2008. The city in the country : Growing alternative food networks in Metropolitan areas, Journal of Rural Studies, vol. 24, p. 231-244.

LAMINE C., 2008. Les AMAP : un nouveau pacte entre producteurs et consommateurs ?, Paris, Éd. Yves Michel, coll. « Société Civile », 163 p.

LE CARO Y., 2007. Les loisirs en espace agricole, l'expérience d'un espace partagé, PUR, coll « Géographie sociale », $431 \mathrm{p}$.

LÉVY J., Lussault M. (dir.), 2000. Logiques d'espaces, esprit des lieux. Géographies à Cérisy, Paris, Belin, coll. « Mappemonde », $351 \mathrm{p}$.

MendRAS H., 1984. La fin des paysans, Paris, Babel/Actes Sud, 436 p.

MATHER C., 1999. Agro-commodity chains, market power and territory : re-regulating South African citrus exports in the 1990's, Geoforum, vol. 30, p. 61-70.

MOULAERT F., SEKIA F., 2003. Territorial Innovation Models, Regional Studies, vol. 37, n 3, p. 289-302. 
PADEL S., 2001. Conversion to Organic Farming : A Typical Example of the Diffusion of an Innovation?, Sociologia Ruralis, vol. 41, $\mathrm{n}^{\circ}$ 1, p. 40-61.

PERRIER-CORNET Ph. (dir.), 2002. Repenser les campagnes, Paris, édition de l'aube, DATAR, bibliothèque des territoires, $287 \mathrm{p}$.

PISANI E., 1994. Pour une agriculture marchande et ménagère, Paris, L'Aube, 191 p.

Poulot M., Rouyres T., 2007. « Refaire campagne en Île-de-France », Norois, n² 202, p. 61-71.

POURSINOFF A., 2008. L'agriculture biologique : Une contribution majeure à l'Écorégion. Pour un plan

d'action, île-de-France, 93 p.

Rallet A, Torre A., 2004. Proximité et localisation, Économie rurale, n 241, p. 25-41.

RALLET A., 2002. L'économie de proximité, Etudes et recherches sur les systèmes Agraires et le développement, Paris, $\mathrm{n}^{\circ} 33$, p. 27-34.

SABATIER B., 2009, Les recompositions territoriales liées á la consommation en Midi-Pyrénées : un enjeu de développement régional, dans HAL-SHS, [http://halshs.archives-ouvertes.fr/docs/00/34/80/49/PDF/ Consommation_territoires.pdf].

TORRE A., CARON A., 2005. Réflexions sur les dimensions négatives de la proximité : le cas des conflits d'usage et de voisinage, Economie et Institutions, n 6-7, p. 183-220.

TREWAVAS A., 2001. Urban myths of organic farming, Nature International weekly journal of science, vol. 410, p. 409-410.

VAN DAM D., 2007. L'agriculture biologique : un autre modèle agricole ou une opportunité économique ?, dans LE CARO Y., MADELINE P., PIERRE G., Agriculteurs et territoires, entre productivisme et exigences territoriales, Rennes, PUR, coll. « Espace et Territoire », p. 352-367.

VANIER M., 2008. Le pouvoir des territoires. Essai sur l'interterritorialité, Paris, Anthropos, 200 p. WEBER M., 1921 (posthume). Économie et société, Tome 1 et 2, Paris, Plon (1971), 410 p. et 448 p. WINTER M., 2003. Embeddedness, the new food economy and defensive localism, Journal of Rural Sociology, vol. 19, $\mathrm{n}^{\circ} 1$, p. 23-32.

\section{NOTES}

1. Ses principes directeurs essaiment parallèlement en Amérique du Nord, en Australie et en Europe dans le courant des années 1970, bien que ses préceptes soient apparus en Suisse au début $\mathrm{du} \mathrm{xx}^{\mathrm{e}}$ siècle.

2. L'agriculture biologique a pour fondement la non-introduction de produits chimiques dans le processus de croissance des ressources alimentaires produites par le travail agricole.

3. Dans le cadre d'un séjour post-doctoral effectué dans l'équipe Proximités de l'UMR INRA SAD-APT 1048, 21 enquêtes ont été réalisées auprès des agriculteurs bio en Île-deFrance, de mai 2009 à mars 2010, soit environ un quart des producteurs biologiques de la région en 2009 (89). En janvier 2010, ils sont 115. Des enquêtes moins formelles auprès des producteurs dans le cadre des paniers fraîcheurs (en gare de Melun et de Sainte-Geneviève-des-Bois) ont été également menées. De plus, des acteurs administratifs ou organisationnels ont été interrogés (le responsable de l'Agriculture 
Biologique de l'Union Européenne, un responsable de l'INAO, un responsable de la FNAB, un responsable du GAB IDF, un responsable de la DRIAF, un responsable du Conseil Régional, un élu du Conseil Régional en charge de l'agriculture biologique). Des enquêtes ont été réalisées dans des Biocoop (Essonne, Paris), des Naturéo (Essonne). Des entretiens téléphoniques avec un négociant bio à Rungis ainsi qu'avec un responsable de la plateforme de Naturéo à Dourdan complètent ces interviews.

4. L'Île-de-France compte douze marchés bio, plus des marchés classiques où les producteurs bio peuvent s'installer.

5. Ils gardent parallèlement des AMAP plus lointaines, notamment celles de Paris qui demeurent encore un débouché conséquent dans le bénéfice des exploitations visitées.

6. Le sud de la Région Île-de-France est un pôle de production des herbes aromatiques, autour de Milly-la-Forêt.

\section{RÉSUMÉS}

La Région Île-de-France a mis en place un programme de soutien pour l'agriculture biologique. Cette forme d'agriculture est conçue en tant qu'alternative au système agricole dominant qui structure les espaces ruraux dans cette région: l'agriculture productiviste. La filière $A B$ qui interdit les intrants chimiques de synthèse dans l'élaboration des produits est un système en construction, dont le développement est favorisé par la volonté politique d'acteurs institutionnels à différentes échelles. Cette filière contribue à l'ordonnancement des espaces du fait des effets de circuits de vente variés, parfois innovants qui contribuent à l'affirmation de nouvelles formes de marché. Toutefois, ces dernières s'appuient également sur des pôles existants. Finalement, cette forme d'agriculture qui implique nombre d'acteurs de natures différentes, impulse de nouvelles formes de coordination et une trajectoire de développement alternative.

"Paris Region" appears as a stakeholder to organic farming on these territories. This form of farming is imagined as an alternative to the agricultural dominant system that structures rural areas in the region: the productivist agriculture. The organic farming which forbids synthetic chemicals in the making of products is under construction. It is facilitated by programmatic of various political and institutional actors, at several scales. This kind of farming organises farming spaces with several types of sale, which offer new marketing forms. However, these last ones also lean on existing poles. Finally, this embeddedness involves different actors who organise themselves to launch a sustainable and alternative development based a new agri-food system.

\section{INDEX}

Mots-clés : agriculture biologique, acteurs

Keywords : organic farming, actors, network 


\section{AUTEURS}

\section{NICOLAS BOIVIN}

UMR SAD-APT Proximités INRA (Institut des sciences et industries du vivant et de l'environnement), 16 rue Claude-Bernard - 75231 Paris Cedex 05,

France,nicolas.boivin@agroparistech.fr

\section{JEAN-BAPTISTE TRAVERSAC}

UMR SAD-APT Proximités INRA (Institut des sciences et industries du vivant et de l'environnement), 16 rue Claude-Bernard - 75231 Paris Cedex 05, France, jeanbaptiste.traversac@agroparistech.fr 